\title{
Regioselectivity Differentiation in Metalations of 3,5-Dichloro-Tertiary versus Secondary Benzamides
}

\author{
Francesca Khani, Tina Fleming, Carleton Collins, Erica Tabakin, Lynn M. Bradley*, \\ David A. Hunt \\ Department of Chemistry, The College of New Jersey, Ewing, USA \\ Email: ${ }^{B}$ Bradley@tcnj.edu
}

Received 1 March 2016; accepted 31 May 2016; published 3 June 2016

Copyright (C) 2016 by authors and Scientific Research Publishing Inc. This work is licensed under the Creative Commons Attribution International License (CC BY). http://creativecommons.org/licenses/by/4.0/

(c) (i) Open Access

\begin{abstract}
Metalation regioslectivity of 3,5-dichlorobenzamides is a function of the type of amide (secondary versus tertiary) used in the sequence. Metalation at the 2-position (adjacent to the carboxamide functional group) occurs when the secondary benzamide is metalated with sec-butyllithium/ TMEDA mediated through complex-induced proximity effects (CIPE) process, whereas metalation with sec-butyllithium/TMEDA occurs exclusively at the 4-position when the tertiary benzamide is used under identical reaction conditions.
\end{abstract}

\section{Keywords}

Directed Ortho-Metalation, Complex-Induced Proximity Effects (CIPE), 3,5-Dichlorobenzamides

\section{Introduction}

Among the most powerful techniques for the introduction of electrophilic functional groups onto an aromatic or heteroaromatic ring system is that of Directed ortho-Metalation (DoM) [1]. Many review articles have been written on the technique over the past three decades describing both synthetic and mechanistic studies [2]-[7]. The importance of this effect has been amply illustrated by adoption of Directed ortho-Metalation as a key synthetic methodology over the past 30 years.

We have previously reported an unexpected regioselectivity observation for the metalation of 3,5-dichloro$N, N$-diethylbenzamide $\underline{1}$ [8]. Addition of sec-butyllithium to a diethyl ether solution of the benzamide at $-78^{\circ} \mathrm{C}$ using benzaldehydes as electrophiles afforded the 4-substituted 3,5-dichlorobenzamides $\underline{\mathbf{3}}$ in moderate to good

Corresponding author.

How to cite this paper: Khani, F., Fleming, T., Collins, C., Tabakin, E., Bradley, L.M. and Hunt, D.A. (2016) Regioselectivity Differentiation in Metalations of 3,5-Dichloro-Tertiary versus Secondary Benzamides. International Journal of Organic Chemistry, 6, 142-146. http://dx.doi.org/10.4236/ijoc.2016.62015 
yields with no detectable product arising from metalation ortho to the tertiary amide (Scheme 1). Possible explanations proposed for this observation involve steric control, electronic control, or a combination of the two. In an effort to shed light on the physicochemical and/or mechanistic basis for this observation, we turned our attention to the metalation of the corresponding 3,5-dichloro- $N$-ethylbenzamide (4). This paper summarizes the difference between the metalation proclivities of 3,5-dichloro tertiary benzamides versus the corresponding secondary benzamides due to differences in the degree of complex-induced proximity effects (CIPE).

\section{Results and Discussion}

For the case of tertiary benzamide systems (Scheme 1), 1.0 - 1.2 molar equivalents of sec-butyllithium is typically employed for metalation, whereas the secondary benzamide requires a minimum of 2.0 molar equivalents of metalating agent, the first equivalent consumed in the generation of the anion $\mathbf{5}$ resulting from the acid-base reaction with the carboxamide functional group (Scheme 2). Through complex-induced proximity effects [4] [9] the metalated carboxamide functional group (5) directs the ortho-metalation through intermolecular complexation with the second equivalent of metalating agent. Since complexation has been shown to be an acidifying event [5], we felt that this chelation control effect may substantially alter any directing effects exerted by the chlorine atoms in the 3- and 5-positions, thereby resulting in metalation at the 2-position relative to the secondary carboxamide functional group for 3,5-dichloro-N-ethylbenzamide 4.

As before, sec-butyllithium was employed as the metalating agent with aromatic aldehydes used as electrophiles. However, in this case we isolated isobenzofuranones of the type 6 resulting from sequential ortho-metalation to form the bis-metalated intermediate 5 followed by nucleophilic addition to the aldehyde and subsequent intramolecular cyclization [10]-[14]. None of the product arising from metalation in the 4-position was detected (Scheme 2).

\section{Conclusion}

This study reveals that there are substantial differences in the degree of complexation (CIPE) of the metalating agent to the secondary versus tertiary carboxamide functional group in poly-substituted aromatic systems bearing other directing groups. These differences can and do play a major role in the regiospecificity of metalation reactions [16]. Studies detailing the behavior of the comparable 3,5-difluoro derivatives will be reported in due course.

\section{Experimental}

General. Tetrahydrofuran was purchased as anhydrous (Fluka) and was stored under a nitrogen blanket and over molecular sieves. Sec-Butyllithium (1.3 M in cyclohexane/hexane) was purchased from Acros Organics or Sigma-Aldrich. ${ }^{1} \mathrm{H}$ NMR $(300 \mathrm{MHz})$ and ${ }^{13} \mathrm{C}$ NMR $(75 \mathrm{MHz})$ data were obtained from a Varian Gemini 300 nuclear magnetic resonance spectrometer referencing tetramethylsilane and utilized $\mathrm{CDCl}_{3}$ lock. IR data were obtained from a Perkin-Elmer Model Spectrum 2000 FT-IR spectrometer. GC/MS data were obtained from an Agilent Technologies 6850 GC/5973 MSD. Microanalyses were performed by Intertek, Whitehouse, NJ. All melting points were obtained from a Mel-Temp heating block apparatus and are uncorrected.

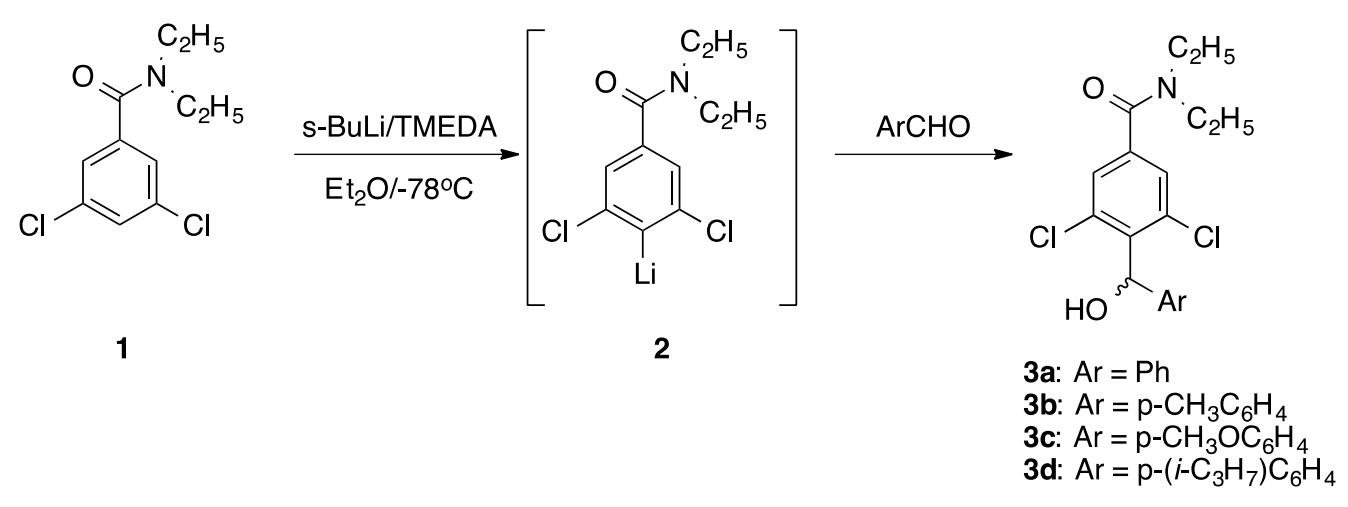

Scheme 1. Para-Metalation/elaboration of a tertiary 3,5-dichlorobenzamide. 
<smiles>CCNC(=O)c1cc(Cl)cc(Cl)c1</smiles>

4

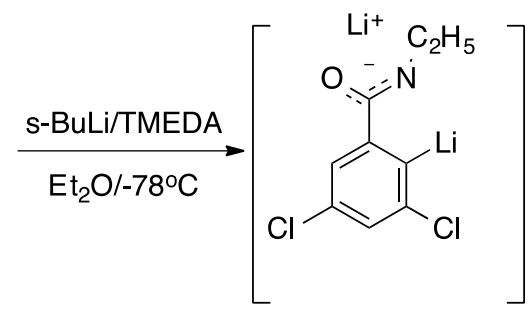

5

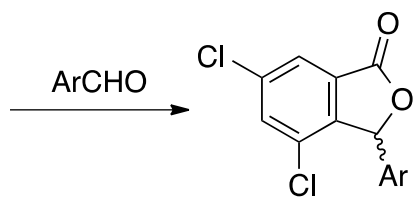

6a: $\mathrm{Ar}=\mathrm{Ph}$

6b: Ar = furan-2-yl

6c: $\mathrm{Ar}=$ thiophen-2-yl

6d: $\mathrm{Ar}=\mathrm{p}-\mathrm{CH}_{3} \mathrm{OC}_{6} \mathrm{H}_{4}$

Scheme 2. Ortho-Metalation/elaboration of a secondary 3,5-dichlorobenzamide.

Metalation of 3,5-Dichloro- $N, N$-Diethylbenzamide: General Reaction Procedure for the Preparation of 3a-d. To a flask containing $246 \mathrm{mg}(1.00 \mathrm{mmol})$ of 3,5-dichloro- $N, N$-diethylbenzamide and $5 \mathrm{~mL}$ of anhydrous diethyl ether was added a solution of TMEDA (1.2 equiv) in $2 \mathrm{~mL}$ of anhydrous diethyl ether. The resulting solution was cooled with magnetic stirring to $-78^{\circ} \mathrm{C}$. To the resulting white suspension was added 1.2 equiv of sec-BuLi in cyclohexane dropwise. The resulting yellow suspension was stirred at $-78^{\circ} \mathrm{C}$ for $30 \mathrm{~min}$, at which point the aryl aldehyde (1.5 equiv) in $2 \mathrm{~mL}$ of anhydrous ether was added drop wise to the reaction mixture. The resulting mixture was allowed to slowly warm to ambient temperature and was stirred an additional $2 \mathrm{~h}$. The mixture was quenched by addition to water. The ether layer was separated, sequentially washed with water and 2 $\mathrm{N} \mathrm{HCl}$, dried $\left(\mathrm{MgSO}_{4}\right)$, filtered, and concentrated. The residue was triturated with petroleum ether to afford the final product.

3,5-Dichloro-N,N-diethyl-4-(hydroxyphenylmethyl)benzamide (3a) was obtained as a colorless solid (152 mg; 54\%): ${ }^{1} \mathrm{H} \mathrm{NMR}\left(300 \mathrm{MHz}, \mathrm{CDCl}_{3}\right) \delta 1.18$ (br m, 3H), 1.26 (br m, 3H), 3.23 (br m, 2H), 3.52 (br m, 2H), $6.65(\mathrm{~s}, 1 \mathrm{H}), 7.26-7.36(\mathrm{~m}, 7 \mathrm{H}) ;{ }^{13} \mathrm{C}$ NMR $(75 \mathrm{MHz}, \mathrm{CDCl} 3) \delta 12.9,14.3,39.6,43.4,72.1,125.5,127.4,128.4$, 135.5, 138.5, 138.9, 141.0, 167.9; IR (KBr) 3411, 2990, 1612, 1541, $1285,1070 \mathrm{~cm}^{-1} ; \mathrm{mp} 129^{\circ} \mathrm{C}-131^{\circ} \mathrm{C}$; MS: $\mathrm{m} / \mathrm{z}=351,353,355\left(\mathrm{M}^{+}\right)$. Anal. Calcd for $\mathrm{C}_{18} \mathrm{H}_{19} \mathrm{NO}_{2} \mathrm{Cl}_{2}: \mathrm{C}, 61.37 ; \mathrm{H}, 5.44 ; \mathrm{N}, 3.98 ; \mathrm{Cl}, 20.13$. Found: $\mathrm{C}$, 61.36; H, 5.37; N, 4.01; Cl, 20.23 .

3,5-Dichloro-N,N-diethyl-4-(hydroxy-p-tolylmethyl)benzamide (3b) was obtained as an off-white solid (161 mg; 55\%): ${ }^{1} \mathrm{H}$ NMR (300 MHz, $\left.\mathrm{CDCl}_{3}\right) \delta 1.18$ (br m, 3H), $1.26(\mathrm{br} \mathrm{m}, 3 \mathrm{H}), 2.35(\mathrm{~s}, 3 \mathrm{H}), 3.23$ (br m, 2H), $3.52(\mathrm{br} \mathrm{m}, 2 \mathrm{H}), 6.61(\mathrm{~s}, 1 \mathrm{H}), 7.16(\mathrm{bs}, 4 \mathrm{H}), 7.36(\mathrm{~s}, 2 \mathrm{H}) ;{ }^{13} \mathrm{C} \mathrm{NMR}\left(75 \mathrm{MHz}, \mathrm{CDCl}_{3}\right) \delta 12.9,14.3,21.2,39.6$, 43.4, 72.2, 125.4, 127.2, 129.1, 135.4, 137.2, 137.9, 138.4, 138.8, 167.9; IR (KBr) 3409, 1612, 1495, 1274, 1070 $\mathrm{cm}^{-1} ; \mathrm{mp} 154^{\circ} \mathrm{C}-155^{\circ} \mathrm{C} ; \mathrm{MS}: \mathrm{m} / \mathrm{z}=365,367,369\left(\mathrm{M}^{+}\right)$. Anal. Calcd for $\mathrm{C}_{19} \mathrm{H}_{21} \mathrm{NO}_{2} \mathrm{Cl}_{2}: \mathrm{C}, 62.30 ; \mathrm{H}, 5.78 ; \mathrm{N}$, $3.82 ; \mathrm{Cl}, 19.36$. Found: C, 61.83; H, 5.74; N, 3.63; Cl, 19.25 .

3,5-Dichloro-N,N-diethyl-4-[hydroxy-(4-methoxyphenyl)methyl)benzamide(3c) was obtained as an offwhite solid (174 mg; 56\%): ${ }^{1} \mathrm{H}$ NMR (300 MHz, $\left.\mathrm{CDCl}_{3}\right) \delta 1.18$ (br m, 3H), 1.26 (br m, 3H), 3.23 (br m, 2H), 3.52 (br m, 2H), 3.81 (s, 3H), $6.58(\mathrm{~s}, 1 \mathrm{H}), 6.87$ (d, J) $8.6 \mathrm{~Hz}, 2 \mathrm{H}), 7.20(\mathrm{~d}, \mathrm{~J}) 8.6 \mathrm{~Hz}, 2 \mathrm{H}), 7.36(\mathrm{~s}, 2 \mathrm{H}) ;{ }^{13} \mathrm{C}$ NMR (75 MHz, $\left.\mathrm{CDCl}_{3}\right)$ ä 12.9, 14.3, 39.6, 43.4, 55.3, 72.1, 113.8, 126.8, 127.2, 132.9, 135.4, 138.5, 138.8, 159.0, 167.9; IR (KBr) 3384, 1607, 1510, 1251, $1050 \mathrm{~cm}^{-1}$; $\mathrm{mp} 117^{\circ} \mathrm{C}-118^{\circ} \mathrm{C}$; MS: $\mathrm{m} / \mathrm{z}=381,383,385\left(\mathrm{M}^{+}\right)$. Anal. Calcd for $\mathrm{C}_{19} \mathrm{H}_{21} \mathrm{NO}_{3} \mathrm{Cl}_{2}: \mathrm{C}, 59.70 ; \mathrm{H}, 5.54 ; \mathrm{N}, 3.66 ; \mathrm{Cl}, 18.55$. Found: C, 59.54; H, 5.44; N, 3.60; Cl, 18.86.

3,5-Dichloro-N,N-diethyl-4-[hydroxy-(4-isopropylphenyl)-methyl)benzamide(3d) was obtained as an offwhite solid (153 mg; 48\%): ${ }^{1} \mathrm{H}$ NMR (300 MHz, $\left.\mathrm{CDCl}_{3}\right) \delta 1.18$ (br m, 3H), 1.26 (br m, 3H), 1.25 (d, J) $3.4 \mathrm{~Hz}$, $6 \mathrm{H}), 2.91(\mathrm{~m}, 1 \mathrm{H}), 3.23(\mathrm{br} \mathrm{m}, 2 \mathrm{H}), 3.52(\mathrm{br} \mathrm{m}, 2 \mathrm{H}), 6.61(\mathrm{~s}, 1 \mathrm{H}), 7.20(\mathrm{bs}, 4 \mathrm{H}), 7.36(\mathrm{~s}, 2 \mathrm{H}),{ }^{13} \mathrm{CNMR}(75 \mathrm{MHz}$, $\left.\mathrm{CDCl}_{3}\right) \delta 12.9,14.3,33.7,39.6,43.4,72.3,125.5,126.5,127.2,135.4,138.2,138.5,138.8,148.1,167.9$; IR (KBr) $3422,2965,1615,1541,1283,1079 \mathrm{~cm}^{-1} ; \mathrm{mp} 154^{\circ} \mathrm{C}-157^{\circ} \mathrm{C} ; \mathrm{MS}: \mathrm{m} / \mathrm{z}=393,395,397\left(\mathrm{M}^{+}\right)$. Anal. Calcd for $\mathrm{C}_{21} \mathrm{H}_{25} \mathrm{NO}_{2} \mathrm{Cl}_{2}: \mathrm{C}, 63.96 ; \mathrm{H}, 6.39 ; \mathrm{N}, 3.55 ; \mathrm{Cl}, 17.98$. Found: $\mathrm{C}, 63.89 ; \mathrm{H}, 6.41 ; \mathrm{N}, 3.48 ; \mathrm{Cl}, 17.67$.

Metalation of 3,5-Dichloro-N-Ethylbenzamide: General Reaction Procedure for the Preparation of 6a-d.

To a $100 \mathrm{~mL}$ three-neck oven-dried flask equipped with a stir bar, nitrogen inlet, and low temperature thermocouple were added the 3,5-dichloro secondary benzamide (1.00 mmol), anhydrous THF (5 mL), and TMEDA ( $290 \mathrm{mg} ; 2.5 \mathrm{mmol})$ in anhydrous THF $(5 \mathrm{~mL})$. The mixture was cooled to $-78^{\circ} \mathrm{C}$ using a dry ice/acetone bath, and sec-butyllithium (2.5 equivalents relative to the starting benzamide) was added while the temperature was 
maintained at or below $-70^{\circ} \mathrm{C}$. The reaction was stirred for $30 \mathrm{~min}$ at which point a solution of 1.5 equivalents of an aromatic aldehyde in anhydrous THF $(2 \mathrm{~mL})$ was added. The reaction was allowed to warm to room temperature overnight under nitrogen and the mixture was quenched with water $(100 \mathrm{~mL})$. The mixture was then extracted with ethyl acetate $(3 \times 40 \mathrm{~mL})$, with occasional use of saturated brine $(35 \mathrm{~mL})$ to alleviate emulsion formation. The organic layer was washed with a solution of saturated ammonium chloride $(40 \mathrm{~mL})$, dried $\left(\mathrm{MgSO}_{4}\right)$, filtered, and concentrated in vacuo to afford the crude product. Purification was achieved by chromatography on silica gel (9:1 hexanes/MTBE) or a combination of trituration/chromatography.

4,6-Dichloro-3-phenylisobenzofuran-1(3H)-one (6a) was isolated as a white solid (chromatography), ${ }^{1} \mathrm{H}$ $\mathrm{nmr}\left(\mathrm{CDCl}_{3}\right): \delta 7.85\left(\mathrm{~d}, \mathrm{~J}_{m-\mathrm{H}}=1.7 \mathrm{~Hz}, 1, \mathrm{ArH}\right), 7.60\left(\mathrm{~d}, \mathrm{~J}_{m-\mathrm{H}}=1.7 \mathrm{~Hz}, 1, \mathrm{ArH}\right), 7.40-7.16(\mathrm{~m}, 5, \mathrm{ArH}), 6.35(\mathrm{~s}$, 1, CHO). ${ }^{13} \mathrm{C} \mathrm{nmr}\left(\mathrm{CDCl}_{3}\right): \delta 168.1,144.9,137.1,135.0,133.8,130.8,130.1,129.9,129.2,128.4,124.4,82.7$; IR: 1776 (lactone) $\mathrm{cm}^{-1}$; mp. $118^{\circ} \mathrm{C}-120^{\circ} \mathrm{C} ; \mathrm{MS}: \mathrm{m} / \mathrm{z}=278,280,282\left(\mathrm{M}^{+}\right)$. Anal. Calcd for $\mathrm{C}_{14} \mathrm{H}_{8} \mathrm{Cl}_{2} \mathrm{O}_{2} ; \mathrm{C}$ 60.24; H, 2.89. Found: C, 60.46; H, 2.71 .

4,6-Dichloro-3-(furan-2-yl)isobenzofuran-1(3H)-one (6b) was isolated as an off-white solid (chromatography), ${ }^{1} \mathrm{H} \mathrm{nmr}\left(\mathrm{CDCl}_{3}\right): \delta 7.83\left(\mathrm{~d}, \mathrm{~J}_{m-\mathrm{H}}=1.5 \mathrm{~Hz}, 1, \mathrm{ArH}\right), 7.63\left(\mathrm{~d}, \mathrm{~J}_{m-\mathrm{H}}=1.5 \mathrm{~Hz}, 1, \mathrm{ArH}\right), 7.38\left(\mathrm{dd}, \mathrm{J}_{\text {furanH2,H3 }}=2.0\right.$ $\left.\mathrm{Hz}, \mathrm{J}_{\text {furanH} 2, \mathrm{H} 4}=0.8 \mathrm{~Hz}, 1, \mathrm{ArH}\right) 6.50\left(\mathrm{dd}, \mathrm{J}_{\text {furanH4,H3 }}=3.3 \mathrm{~Hz}, \mathrm{~J}_{\text {furanH4,H2}}=0.6 \mathrm{~Hz}, 1, \mathrm{ArH}\right), 6.40(\mathrm{~s}, 1, \mathrm{CHO}), 6.39$

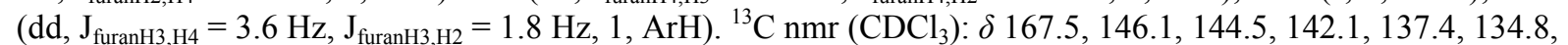
130.8, 130.2, 124.5, 112.6, 111.1, 74.8; IR: 1778 (lactone) $\mathrm{cm}^{-1}$; mp. $115.5^{\circ} \mathrm{C}-117^{\circ} \mathrm{C} ; \mathrm{MS}: \mathrm{m} / \mathrm{z}=268,270,272$ $\left(\mathrm{M}^{+}\right)$. Anal. Calcd for $\mathrm{C}_{12} \mathrm{H}_{6} \mathrm{Cl}_{2} \mathrm{O}_{3} ; \mathrm{C}, 53.56 ; \mathrm{H}, 2.25$. Found: $\mathrm{C}, 53.27 ; \mathrm{H}, 2.25$.

4,6-Dichloro-3-(thiophen-2-yl)isobenzofuran-1(3H)-one (6c) was isolated as an off-white solid (trituration/ chromatography), ${ }^{1} \mathrm{H} n m r\left(\mathrm{CDCl}_{3}\right): \delta 7.84\left(\mathrm{~d}, \mathrm{~J}_{m-\mathrm{H}}=1.8 \mathrm{~Hz}, 1, \mathrm{ArH}\right), 7.64\left(\mathrm{~d}, \mathrm{~J}_{m-\mathrm{H}}=1.5 \mathrm{~Hz}, 1, \mathrm{ArH}\right), 7.38(\mathrm{dd}$, $\left.\mathrm{J}_{\mathrm{H} 2, \mathrm{H} 3}=5.4 \mathrm{~Hz}, \mathrm{~J}_{\mathrm{H} 2, \mathrm{H} 4}=0.9 \mathrm{~Hz}, 1, \mathrm{ArH}\right), 7.14\left(\mathrm{dd}, \mathrm{J}_{\mathrm{H} 4, \mathrm{H} 3}=3.6 \mathrm{~Hz}, \mathrm{~J}=1.2 \mathrm{~Hz}, 1, \mathrm{ArH}\right), 7.01\left(\mathrm{dd}, \mathrm{J}_{\mathrm{H} 3, \mathrm{H} 2}=5.1 \mathrm{~Hz}\right.$, $\left.\mathrm{J}_{\mathrm{H} 3, \mathrm{H} 4}=3.6 \mathrm{~Hz}, 1, \mathrm{ArH}\right), 6.63(\mathrm{~s}, 1, \mathrm{CHO}) .{ }^{13} \mathrm{C} \mathrm{nmr}\left(\mathrm{CDCl}_{3}\right): \delta 167.3,144.1,137.4,136.4,135.0,130.9,129.4$, 128.2, 127.3, 127.1, 124.4, 77.2; IR: 1774 (lactone) $\mathrm{cm}^{-1}$; mp. $107.5^{\circ} \mathrm{C}-109.5^{\circ} \mathrm{C} ; \mathrm{MS}: \mathrm{m} / \mathrm{z}=284,286,288\left(\mathrm{M}^{+}\right)$. Anal. Calcd for $\mathrm{C}_{12} \mathrm{H}_{6} \mathrm{Cl}_{2} \mathrm{O}_{2} \mathrm{~S} ; \mathrm{C}, 50.54 ; \mathrm{H}, 2.12$. Found: C, 50.83; H, 2.31 .

4,6-Dichloro-3-(4-methoxyphenyl)isobenzofuran-1(3H)-one (6d) was isolated as a white solid (trituration/chromatography); ${ }^{1} \mathrm{H} \mathrm{nmr}\left(\mathrm{CDCl}_{3}\right): \delta 7.84\left(\mathrm{~d}, \mathrm{~J}_{m-\mathrm{H}}=1.5 \mathrm{~Hz}, 1, \mathrm{ArH}\right), 7.59\left(\mathrm{~d}, \mathrm{~J}_{m-\mathrm{H}}=1.8 \mathrm{~Hz}, 1, \mathrm{ArH}\right), 7.10$ $\left(\mathrm{d}, \mathrm{J}_{\mathrm{a}, \mathrm{b}}=9.0 \mathrm{~Hz}, 2, \mathrm{ArH}\right), 6.86\left(\mathrm{~d}, \mathrm{~J}_{\mathrm{b}, \mathrm{a}}=9.0 \mathrm{~Hz}, 2, \mathrm{ArH}\right), 6.32(\mathrm{~s}, 1, \mathrm{CHO}), 3.79\left(\mathrm{~s}, 3, \mathrm{OCH}_{3}\right) .{ }^{13} \mathrm{C} \mathrm{nmr}_{\left(\mathrm{CDCl}_{3}\right): \delta}$ 169.0, 160.8, 145.0, 136.9, 134.8, 130.7, 129.8, 129.7, 125.6, 124.2, 114.4, 82.4, 55.5; IR: 1773 (lactone) $\mathrm{cm}^{-1}$; mp. $107.5^{\circ} \mathrm{C}-109^{\circ} \mathrm{C} ;$. MS: m/z $=308,310,312\left(\mathrm{M}^{+}\right)$. Anal. Calcd for $\mathrm{C}_{15} \mathrm{H}_{10} \mathrm{Cl}_{2} \mathrm{O}_{3} ; \mathrm{C}, 58.28 ; \mathrm{H}, 3.26$. Found: C, $58.30 ; \mathrm{H}, 3.31$.

\section{Acknowledgements}

We would like to thank the Department of Chemistry, The College of New Jersey for partial financial support of this work.

\section{References}

[1] El-Hiti, G.A., Smith, K., Hegazy, A.S., Alshammari, M.B. and Masmali, A.M., ARKIVOC (Gainesville, FL, United States) (2015) For a Comprehensive Recent Review of Directed Ortho-Metalation. 4Spec.Issue, 19-47/1-19-47/30.

[2] Snieckus, V. (1990) Directed Ortho Metalation. Tertiary Amide and O-Carbamate Directors in Synthetic Strategies for Polysubstituted Aromatics. Chemical Reviews, 90, 879-933. http://dx.doi.org/10.1021/cr00104a001

[3] Epsztajn, J., Jozwiak, A. and Szczesniak, A.K. (2006) Secondary Amides as Ortho-Directed Metallation Groups for Arenes: A Useful Construction Way of the Polysubstituted Aromatic and Heteroaromatic Systems. Current Organic Chemistry, 10, 1817-1848. http://dx.doi.org/10.2174/138527206778249883

[4] Beak, P. and Meyers, A.I. (1986) Stereo- and Regiocontrol by Complex Induced Proximity Effects: Reactions of Organolithium Compounds. Accounts of Chemical Research, 19, 356-363. http://dx.doi.org/10.1021/ar00131a005

[5] Beak, P. and Snieckus, V. (1982) Directed Lithiation of Aromatic Tertiary Amides: An Evolving Synthetic Methodology for Polysubstituted Aromatics. Accounts of Chemical Research, 15, 306-312. http://dx.doi.org/10.1021/ar00082a002

[6] Schlosser, M. (2005) The $2 \times 3$ Toolbox of Organometallic Methods for Regiochemically Exhaustive Functionalization. Angewandte Chemie International Edition, 44, 376-393. http://dx.doi.org/10.1002/anie.200300645

[7] Campos, K.R. (2007) Direct Sp3 C-H Bond Activation Adjacent to Nitrogen in Heterocycles. Chemical Society Reviews, 36, 1069-1084. http://dx.doi.org/10.1039/B607547A 
[8] Demas, M., Javadi, G.J., Bradley, L.M. and Hunt, D.A. (2000) Metalation of a 3,5-Dichloro-Tertiary Benzamide. An Unusual Regioselectivity Observation. The Journal of Organic Chemistry, 65, 7201-7202. http://dx.doi.org/10.1021/jo000160t

[9] Marna, C., Whisler, M.C., MacNeil, S., Snieckus, V. and Beak, P. (2004) Angewandte Chemie International Edition, 43, 2206-2225.

[10] Fraser, R.R., Bresse, M. and Mansour, T.S. (1983) Ortho Lithiation of Monosubstituted Benzenes: A Quantitative Determination of pKa Values in Tetrahydrofuran. Journal of the American Chemical Society, 105, 7790-7791. http://dx.doi.org/10.1021/ja00364a078

[11] Shatenshtein, A.I. (1962) Mechanism Study of the Protophilic Substitution of Hydrogen in Aromatic Compounds by Means of Hydrogen Isotope Exchange with Liquid Ammonia. Tetrahedron, 18, 95-106. http://dx.doi.org/10.1016/0040-4020(62)80029-5

[12] Shirley, D.A. and Hendrix, J.P. (1968) Steric Effects in the Metalation of Some Aromatic Substrates with Alkyllithium Reagents. Journal of Organometallic Chemistry, 11, 217-226. http://dx.doi.org/10.1016/0022-328X(68)80044-0

[13] Shirley, D.A., Harmon, T.E. and Chemg, C.F. (1974) Some Observations Pertaining to the Mechanism of Metalation of Aromatic Substrates with Alkyllithium Reagents. Journal of Organometallic Chemistry, 69, 327-344. http://dx.doi.org/10.1016/S0022-328X(00)89736-3

[14] Slocum, D.W. and Koonsvitsky, B.P. (1973) Directed Metalation Reactions. III. Contribution of Oxygen Coordination in the Lithiation of O-Tert-Butylanisole. The Journal of Organic Chemistry, 38, 1675-1677. http://dx.doi.org/10.1021/jo00949a011

[15] Bradley, L.M., Collins, C.G., Tabakin, E.R. and Hunt, D.A. (2010) Expedient Preparation of 4,6-Dihalo-3-Arylisobenzofuran-1(3H)-Ones from 3,5-Dihalo-N-Ethylbenzamides. Organic Preparations and Procedures International, 42, 187-190. http://dx.doi.org/10.1080/00304941003727045

[16] Beak, P. and Brown, R.A. (1982) The Tertiary Amide as an Effective Director of Ortho Lithiation. The Journal of Organic Chemistry, 47, 34-46. http://dx.doi.org/10.1021/jo00340a008 